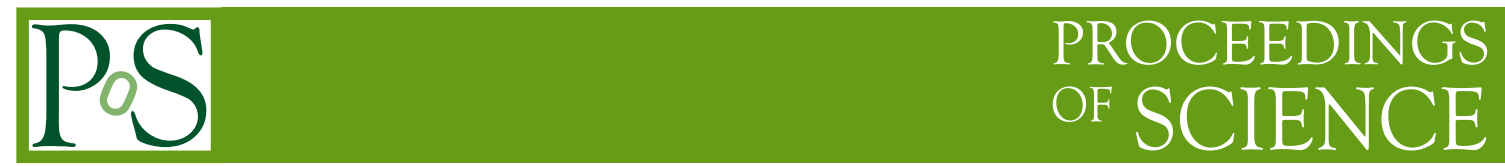

\title{
Effective operators in top physics
}

\author{
Juan Antonio Aguilar-Saavedra*t \\ University of Granada \\ E-mail: jaas@ugr.es
}

Top interactions beyond the Standard Model can be conveniently described in the framework of gauge-invariant effective operators. We briefly review the general form of the fermion-fermiongauge boson and fermion-fermion-Higgs interactions arising from dimension- 6 operators, focusing on the top quark. We discuss in detail an expansion in powers of $1 / \Lambda^{2}$, where $\Lambda$ is the new physics scale, to consistently calculate observables in terms of top anomalous couplings.

35th International Conference of High Energy Physics

July 22-28, 2010

Paris, France

* Speaker.

$\dagger$ This work has been partially supported by a MICINN Ramón y Cajal contract and by projects FPA2006-05294 (MICINN), FQM 101 and FQM 437 (Junta de Andalucía), CERN/FP/83588/2008 (FCT), and MRTN-CT-2006-035505 (European Union). 


\section{Introduction}

One of the main motivations for detailed studies of the top quark properties is to search for indirect effects of new physics not directly observable. Heavy physics above the electroweak scale can be parameterised using an effective Lagrangian

$$
\mathscr{L}^{\mathrm{eff}}=\sum \frac{C_{x}}{\Lambda^{2}} O_{x}+\ldots,
$$

where $O_{x}$ are dimension-6 operators invariant under the Standard Model (SM) gauge symmetry, $\Lambda$ the new physics scale and the dots stand for dimension- 8 and higher-order operators, usually ignored. Dimension-6 operators were classified in [1] and an operator basis was given, which can be used to parameterise any new physics contribution up to higher orders. Later, it has been found [2, 3] that some of these operators are actually redundant and can be dropped from the list. This simplification is very useful for phenomenology, because it implies that (i) the most general fermion-fermion-gauge boson interactions arising from dimension-6 operators contains at most $\gamma^{\mu}$ and $\sigma^{\mu v} q_{v}$ terms, where $q$ is the boson momentum; (ii) the most general fermion-fermion-Higgs interactions only contain scalar and pseudo-scalar terms. In the particular case of the top quark we have, for example, the most general $W t b$ vertex

$$
\begin{aligned}
\mathscr{L}_{W t b}= & -\frac{g}{\sqrt{2}} \bar{b} \gamma^{\mu}\left(V_{L} P_{L}+V_{R} P_{R}\right) t W_{\mu}^{-} \\
& -\frac{g}{\sqrt{2}} \bar{b} \frac{i \sigma^{\mu v} q_{v}}{M_{W}}\left(g_{L} P_{L}+g_{R} P_{R}\right) t W_{\mu}^{-}+\text {h.c. },
\end{aligned}
$$

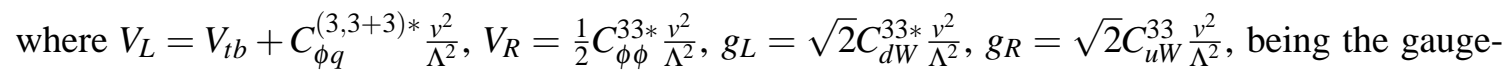
invariant operators

$$
\begin{array}{ll}
O_{\phi q}^{(3,3+3)}=\frac{i}{2}\left[\phi^{\dagger}\left(\tau^{I} D_{\mu}-\overleftarrow{D}_{\mu} \tau^{I}\right) \phi\right]\left(\bar{q}_{L 3} \gamma^{\mu} \tau^{I} q_{L 3}\right), & O_{\phi \phi}^{33}=i\left(\tilde{\phi}^{\dagger} D_{\mu} \phi\right)\left(\bar{t}_{R} \gamma^{\mu} b_{R}\right) \\
O_{d W}^{33}=\left(\bar{q}_{L 3} \sigma^{\mu v} \tau^{I} b_{R}\right) \phi W_{\mu v}^{I}, & O_{u W}^{33}=\left(\bar{q}_{L 3} \sigma^{\mu v} \tau^{I} t_{R}\right) \tilde{\phi} W_{\mu \nu}^{I}
\end{array}
$$

in standard notation. Were it not for this simplification, the $W t b$ vertex would contain 6 additional Lorentz structures, with 6 more parameters arising from 5 (redundant) operators. Additional details can be found in Refs. [3].

One may always ask about precision electroweak data and constraints on these operators because, being the top quark the $\mathrm{SU}(2)_{L}$ partner of the $b$ quark, gauge invariant operators containing the $t_{L}$ field automatically involve $b_{L}$. For example, the $Z b_{L} b_{L}$ vertex, well measured at LEP, receives corrections from effective operators

$$
\mathscr{L}_{Z b_{L} b_{L}}=-\frac{g}{2 c_{W}} \bar{b}_{L} \gamma^{\mu}\left[-1+\left(C_{\phi q}^{(3,3+3)}+C_{\phi q}^{(1,3+3)}\right) \frac{v^{2}}{\Lambda^{2}}+\frac{2}{3} s_{W}^{2}\right] b_{L} Z_{\mu}
$$

where $O_{\phi q}^{(3,3+3)}$ is the operator producing a deviation $\delta V_{L} \equiv V_{L}-V_{t b}$ in the Wtb vertex, and $O_{\phi q}^{(1,3+3)}=$ $\frac{i}{2}\left(\phi^{\dagger} \overleftrightarrow{D^{\mu}} \phi\right)\left(\bar{q}_{L 3} \gamma^{\mu} q_{L 3}\right)$. Does this imply that $C_{\phi q}^{(3,3+3)}$ is small, because fine-tuning with $C_{\phi q}^{(1,3+3)}$ (so as to keep agreement with LEP data) is unnatural? The answer is no. For example, mixing with a

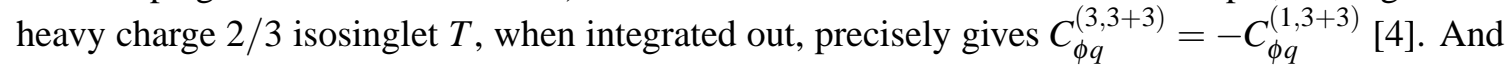


it is clear that it must be so, because the mixing of $T$ with the SM charge $2 / 3$ mass eigenstates does not affect the down sector, where the GIM mechanism is still at work. When written in the language of effective operators, this is just the relation $C_{\phi q}^{(3,3+3)}=-C_{\phi q}^{(1,3+3)}$. Detailed analyses of the effects on $B$ physics of $W t b$ anomalous couplings imply that $V_{R}$ and $g_{L}$ (that is, $C_{\phi \phi}^{33}$ and $C_{d W}^{33}$ ) are very constrained if no other new physics is present [5]. But discarding operators merely because they contain terms with two $b$ fields is, as we can see, too naive and restrictive.

\section{The leading order approximation}

The anomalous couplings $\delta V_{L}, V_{R}, g_{L}, g_{R}$ in Eq. (1.2) are proportional to the scale ratio $v^{2} / \Lambda^{2}$, and thus some expansion in powers of $1 / \Lambda^{2}$ seems adequate. In general, the interference terms between SM amplitudes and dimension- 6 operators are proportional to $1 / \Lambda^{2}$, and the pure dimension6 operator corrections to $1 / \Lambda^{4}$. One might then naively consider keeping only the linear $1 / \Lambda^{2}$ terms, which would give the "leading" corrections, and drop the quadratic $1 / \Lambda^{4}$ ones. But this " $1 / \Lambda^{2}$ " approximation is far too restrictive, and does not allow to explore many new physics effects. Indeed, operators which do not interfere with the SM are the ones producing genuine new physics effects, beyond corrections to SM processes. One well-known example is given by top flavour-changing neutral processes, extremely suppressed in the SM, which are of order $1 / \Lambda^{4}$. These processes, absent in the SM, could be visible for this reason even if suppressed by $1 / \Lambda^{4}$.

For the $W t b$ vertex, in the $m_{b}=0$ limit (we will consider a non-zero $b$ mass later) only the operators generating $\bar{b}_{L} t_{L}$ and $\bar{b}_{L} t_{R}$ terms interfere with the SM amplitude. Then, the $1 / \Lambda^{2}$ approximation leads us to keep only the corrections proportional to $\delta V_{L}$ and $g_{R}$ (see Table 1 ). This is unfortunate, because we lose the possibility of exploring $b$ chirality-breaking effects. It is well

$\begin{array}{ccc} & 1 / \Lambda^{2} & 1 / \Lambda^{4} \\ \bar{b}_{L} \gamma^{\mu} t_{L} & \delta V_{L} & \left(\delta V_{L}\right)^{2}+\operatorname{dim}-8 \bar{b}_{L} t_{L} \\ \bar{b}_{R} \gamma^{\mu} t_{R} & - & \left(V_{R}\right)^{2} \\ \bar{b}_{R} \sigma^{\mu v} t_{L} & - & \left(g_{L}\right)^{2} \\ \bar{b}_{L} \sigma^{\mu v} t_{R} & g_{R} & \left(g_{R}\right)^{2}+\operatorname{dim}-8 \bar{b}_{L} t_{R}\end{array}$

Table 1: Corrections to observables from $W t b$ anomalous couplings, for $m_{b}=0$.

known that in SM top decays the fraction $F_{+}$of $W$ bosons produced with helicity +1 is zero in the $m_{b}=0$ limit. The reason is that for the SM $W t b$ vertex only left-handed $b$ quarks are produced in the decay, and then angular momentum conservation forbids $W$ bosons with helicity +1 . (Including a $b$ quark mass $m_{b}=4.8 \mathrm{GeV}$ the fraction of positive helicity $W$ bosons is $F_{+}=0.00359$.) However, the anomalous couplings $\delta V_{L}, g_{R}$ still involve left-handed $b$ quarks (otherwise they would not interfere with the SM amplitude) and their presence does not affect $F_{+}$. This can be seen in Fig. 1, taken from Ref. [6]. The (quadratic) corrections from anomalous couplings $V_{R}, g_{L}$ lead to dramatic departures from the SM prediction while a non-zero $g_{R}$ makes no difference. The sensitivity to these deviations in top decays at LHC is excellent [7] precisely because $F_{+}$is tiny within the SM, and in the $1 / \Lambda^{2}$ approximation such effects are lost.

Let us then discuss how the $1 / \Lambda^{2}$ approximation may be refined. As we see from Table 1 , for $\bar{b}_{L} t_{L}$ and $\bar{b}_{L} t_{R}$ terms the linear corrections are of order $1 / \Lambda^{2}$ while quadratic ones, as well as 

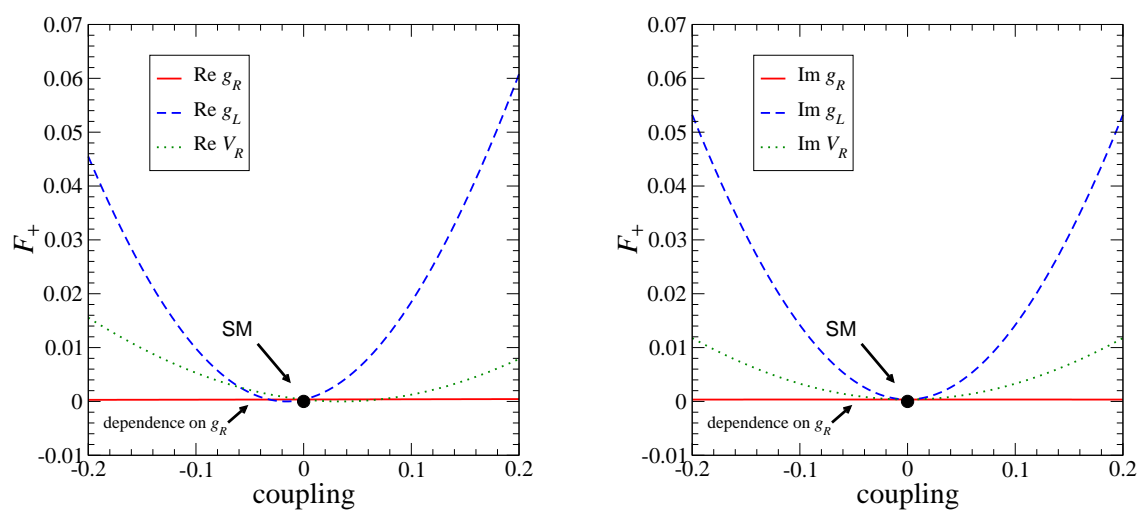

Figure 1: Dependence of the helicity fraction $F_{+}$on $W t b$ anomalous couplings, for $V_{L}=1$.

those from dimension- 8 operators with $\bar{b}_{L} t_{L}, \bar{b}_{L} t_{R}$ fields, are of order $1 / \Lambda^{4}$. For $\bar{b}_{R} t_{R}, \bar{b}_{R} t_{L}$ fields the leading corrections are the quadratic $1 / \Lambda^{4}$ ones $\left(V_{R}\right)^{2},\left(g_{L}\right)^{2}$, while dimension- 8 operators would give non-interfering corrections of order $1 / \Lambda^{8}$. It is then consistent to keep for each $t, b$ chirality the lowest-order terms. (Sub-leading $\left(\delta V_{L}\right)^{2},\left(g_{R}\right)^{2}$ terms cause no harm and may be kept or discarded at will.) This is what we denote as the "leading order approximation". When the $b$ quark mass is included, interferences of $\bar{b}_{R} t_{R}$ and $\bar{b}_{R} t_{L}$ operators are suppressed by $\left(m_{b} / m_{t}\right) 1 / \Lambda^{2}$, and their effect is of the same order as the quadratic terms [7]. Complete calculations of several top decay observables, keeping quadratic terms as well as the $b$ quark mass, have been performed in Refs. [6, 不, and for single top cross sections in Ref. [8].

\section{References}

[1] W. Buchmuller and D. Wyler, Effective Lagrangian analysis of new interactions and flavor conservation, Nucl. Phys. B 268 (1986) 621.

[2] B. Grzadkowski, Z. Hioki, K. Ohkuma and J. Wudka, Probing anomalous top-quark couplings induced by dim.6 operators at photon colliders, Nucl. Phys. B 689 (2004) 108

[3] J. A. Aguilar-Saavedra, A minimal set of top anomalous couplings, Nucl. Phys. B 812 (2009) 181; A minimal set of top-Higgs anomalous couplings, Nucl. Phys. B 821 (2009) 215.

[4] F. del Aguila, M. Perez-Victoria and J. Santiago, Effective description of quark mixing, Phys. Lett. B 492 (2000) 98; Observable contributions of new exotic quarks to quark mixing, JHEP 0009 (2000) 011.

[5] B. Grzadkowski and M. Misiak, Anomalous Wtb coupling effects in the weak radiative B-meson decay, Phys. Rev. D 78 (2008) 077501.

[6] J. A. Aguilar-Saavedra and J. Bernabeu, $W$ polarisation beyond helicity fractions in top quark decays, Nucl. Phys. B 840 (2010) 349.

[7] J. A. Aguilar-Saavedra, J. Carvalho, N. F. Castro, A. Onofre and F. Veloso, Probing anomalous Wtb couplings in top pair decays, Eur. Phys. J. C 50 (2007) 519; ATLAS sensitivity to Wtb anomalous couplings in top quark decays, Eur. Phys. J. C 53 (2008) 689.

[8] J. A. Aguilar-Saavedra, Single top quark production at LHC with anomalous Wtb couplings, Nucl. Phys. B 804 (2008) 160. 